\title{
Cervical ectopic pregnancy mimicking missed abortion
}

\author{
E Saha1, AK Paul2,
}

\begin{abstract}
Summary
Cervical pregnancy is a rare variety of Ectopic pregnancy. It is a life threatening form of ectopic pregnancy. The diagnosis of cervical pregnancy can be missed by an unsuspecting mind inspite of the routine use of first trimester Ultrasonography. Careful evaluation is necessary for immediate proper management of such case.
\end{abstract}

Bang Med J (Khulna) 2011; 44 : 28-30

\begin{abstract}
Introduction
Cervical ectopic pregnancy is the implantation of a pregnancy in the endocervical canal. The incidence of cervical ectopic pregnancy is reported to be between 1 in 10,000 to 16,000 deliveries. 1 Cervical ectopic represent less than $1 \%$ of all ectopic pregnancies. 2 Diagnosis and treatment of cervical ectopic pregnancy has changed dramatically in the last two decades. Before 1980, the diagnosis commonly was made when dilatation and curettage for presumed incomplete abortion resulted in unexpected hemorrhage. Now cervical ectopic pregnancy is commonly diagnosed on a first trimester ultrasound examination. Although the advent of sonography has made the diagnosis more accurate, it is still a problem in developing countries. Clinically it is often mistaken for inevitable or missed abortion.3 Sonologist may be mistaken as incomplete or missed abortion in lower uterine cavity. Recently we encountered a case of cervical pregnancy of a thirty five year old lady with a history of ten weeks amenorrhea, slight per vaginal bleeding and moderate lower abdominal pain for fifteen days. In our clinical experience, this is an extreme rare disorder and we were interested to report this case.
\end{abstract}

\section{Case report}

A thirty five years old lady (para 2+2) presented with amenorrhea of ten weeks with history of slight per vaginal bleeding for fifteen days and moderate lower abdominal pain for three days. Her pregnancy test was positive at six weeks of amenorrhea. She was treated with antipsychotic drug. On examination her vitals were stable and mild pallor was present, supra pubic \& both iliac fossa were moderately tender. On per vaginal examination, cervix feels soft, slightly distended, mild old blood- stained discharge present, os closed, size of uterus about eight weeks of pregnant size which was tender, Fresh blood comes out during bimanual examination. Clinically missed abortion suspected.
Trans abdominal sonography shows about $4.8 \times 4.0 \mathrm{~cm}$ mixed ecogenic structure present on the lower uterine segment suggestive of missed abortion (fig-1). The patient was taken for dilatation, evacuation and curettage under general anesthesia. As soon as dilator was put in the cervical canal patient started bleeding profusely. Old product of conception was brought out with sponge holding forceps from endocervical canal which was adherent tightly on right lateral wall of cervix. Implantation of placenta in the wall of endocervical canal was diagnosed. Uterine cavity was empty but bleeding continued. For stopping hemorrhage bi-manual compression and oxytocic drugs were given. But bleeding did not control.

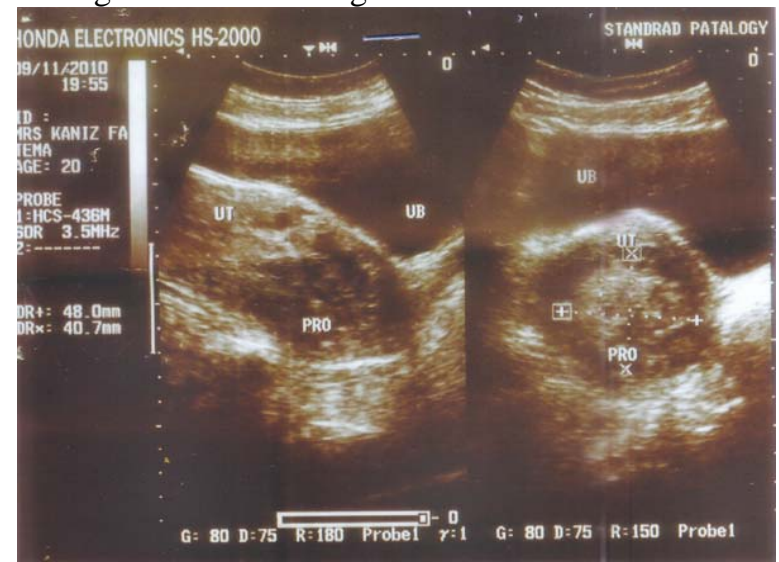

Fig-1: Trans Abdominal Sonography showing product of conception in lower uterine segment \& empty uterine cavity.

Then tight packing of cervical canal and vagina with gauge and pad. Patient was transfused two units of blood. After one hour vaginal packs were soaked with blood. Then patient was shifted again to operation theatre for emergency laparotomy with the informed consent for hysterectomy. At laparotomy the uterus was normal sized but the cervix was grossly dilated \& sign of placental attachment was present. A total abdominal hysterectomy was performed. The patient received seven units of blood. Post operatively, she recovered well and was discharged on the 7 th post operative day. 


\section{Discussion}

Cervical ectopic pregnancy (CEP) is extremely rare. It was first described in 1817 and it was so named in 1860. In 1911, Rubin in his case report established diagnostic criteria for CEP (Cervical ectopic pregnancy), 4 close attachment of placenta to cervix, cervical glands present opposite the implantation site, placental location below uterine vessel insertion and no fetal elements in the uterine corpus. Ultra Sonography preferably Trans vaginal Sonography (TVS) and Beta-HCG estimation permit early and accurate diagnosis of CEP.5-8 Ultrasound criteria for CEP are (1) echo free uterine cavity (2) decidual transformation of the endometrium with dense echo structure (3) hour glass uterine shape (4) ballooned cervical canal (5) gestational sac in the endocervix (6) placental tissue in the cervical canal (7) closed internal os. The serum Beta HCG concentration doubles up in 48 hours in normal pregnancy. In abnormal pregnancy including ectopic pregnancy, BHCG level do not increase at this rate.

An actual increase in the incidence of cervical ectopic pregnancy may be attributed to an increased prevalence of women with history of uterine curettage and the use of interventional treatment in women with infertility. Often patients with cervical pregnancy are misdiagnosed as complications of intrauterine pregnancy by the attending doctor and the correct diagnosis only made after further reviews.

Vineeta Gupta et a19 reported two cases of ectopic cervical pregnancy, in which first case was wrongly diagnosed as missed abortion and D\&E was tried but it led to profuse bleeding then total abdominal hysterectomy was done as life saving measure. 2nd case was wrongly diagnosed as missed abortion \& D\&E led to profuse bleeding, then bleeding stopped with ballooning of Foley's catheter in cervical canal for tamponade.

Sivalingam et al10 reported three cases of cervical pregnancy with variable clinical presentation and managed by different modalities of treatment. Jacob et al11 reported an ectopic cervical pregnancy which presented as missed abortion that diagnosis was only made at surgical evacuation during which the sudden severe hemorrhage could only be controlled by emergency hysterectomy.

LP Banu, et a112 reported an ectopic cervical pregnancy, which was wrongly diagnosed as molar pregnancy or choriocarcinoma, methotrexate were given. Then patient started bleeding profusely and emergency hysterectomy was done for life saving. After operation naked eye \& histopathological examination confirmed cervical ectopic pregnancy.

In this study, the case was wrongly diagnosed as missed abortion and D, E \& C was tried but it led to profuse bleeding. Vaginal and cervical canal were tightly packed with gauze pack and sanitary pad which was not sufficient for stoping bleeding. Total abdominal hysterectomy was done. Hysterectomy could be avoided with the use of a large Foley catheter balloon.13

Management options of cervical ectopic pregnancy14 are (1) Tamponade- cervical, or vaginal packing \& Foley balloon- they are not definitive therapy, only stabilize for transfer. (2) Blood supply reduction- cervical circlage, angiographic embolization \& large vessel ligation- these preserve fertility and auxiliary technique to prevent or control hemorrhage. (3) Trophoblast excision a) Dilation and curettage of high risk of hemorrhage- so in combination with temponade and or blood supply reduction to preserve fertility, b) Hysteroscopic resection c) Hysterectomy. (4) Intra-amniotic injection with potassium chloride or methotrexate- requires expertise \& preserve fertility. (5) Systemic chemotherapy intramuscular methotrexate and other chemo - therapeutic agents.

There were some case reports of successful treatment with methotrexate.15 Creming and Feldstein16 reported successful conservative management with selective right hypogastric and left uterine artery embolization followed by suction evacuation. Successful tomponade technique was described by Van Meersche et al.17 But in the presence of profuse hemorrhage, early decision to radical treatment may produce good maternal out come.11

Cervical ectopic pregnancy remains an uncommon entity. Clinical awareness, use of an endovaginal ultrasound \& BHCG estimation in the first trimester may help in proper diagnosis. Early accurate detection of such life threatening condition is important for better management.

\section{Reference}

1. S. Hemmodi T. S, Shylasree, K. Bhal, A Rees. Cervical pregnancy, The internet journal of Gynecology and obstetrics, 2005; 4; 1.

2. Ushakov FB, Elchalal U, Aceman PT, Schenker JG. Cervical pregnancy: Past and future, Obstet Gynecol Surv 1997;48:130-132

3. Hingorani SR, Parulekar SV, Ratnam Kl. Isthmico-cervical ectopic pregnancy following cesarean section, J Postgrad Med 1994: 40:33-35.

4. Rubin IC. Cervical pregnancy, Surgical Gynecology obs, 1911; 13: 625-633.

5. Hsu JJ. Chiu TH, Lai IM, Soong YK. Methotrexate treatment of cervical pregnancy with different clinical parameters; a report of three cases, J Reprod Med 1995; 40:246-250.

6. Dotters DJ, Katz VL, Kuller JA, McCoy MC. Successful treatment of cervical pregnancy with a single low dose methotrexate regimen, Europe Journal obstet gynecol Reprod biol 1995; 60 : 187-189.

7. Jonathan S. Berek, MD, MMSC. Editor, In Novak's gynecology, 13th ed,USA, Lippincott Williams and Wilkins publications 2002.

8. M Sivasuriya. Obstetrics and gynecology for post graduates, volume-2; Anna Salai, Madras, Orient- Longman ltd. Publication- 1995, 160.

9. Vineeta G. Rajiv A. Neeta B, Anju N, Archana T, Aruna R. Narendra M. Cervical ectopic pregnancy- Case reports and 
management modalities; Journal of SAFOG, JanuaryApril 2010; 2 : 77-79.

10. N Sivalingam, FK Mak. Delayed diagnosis of cervical pregnancy: Management options, Singapore Med J 2000; 42 ; 12 : 599-601.

11. Jacob A unuigob, Tasreem M Malik. Cervical pregnancy presenting as a missed abortion, Annals of Soudi Medicine 1997, 11; 4 : 462.

12. LP Banu, S Chowdhury, K Begum, F Islam, S Tasnim. Cervical ectopic pregnancy: Case report; J Bangladesh coll phys surg 2007; 25 : 92-94.

13. Victor A Hurley, Norman A Beischer. Cervical pregnancy: Hysterectomy avoided with the use of large Foley catheter balloon. The Australian and New
Zealand Journal of Obs and Gyne 1998; 28; 3 : 230-32.

14. Lawrence M, Leeman MD, MPH. Cervical ectopic pregnancy Arch Fam Med 2000; 9 : 72-77.

15. S Chew, C Anandakumer. Singapore Med 2001; 42 : 11; 537-539.

16. Feichtinger $\mathrm{W}$, Kemeter $\mathrm{P}$. Conservative treatment of ectopic pregnancy by transvaginal aspiration under sonographic control and Methotrexate injection. Lancet 1987; $1: 381$.

17. Van de Meerssche M, Verdonk P, Jacquemyn Y, Serreyn R and Gerris J. Cervical pregnancy: three case reports and a view of the literature, Hum Repro 1995; 10 : 1850-5. 